\title{
Cricoarytenoid Joint Fixation as a presenting feature of Juvenile Rheumatoid Arthritis
}

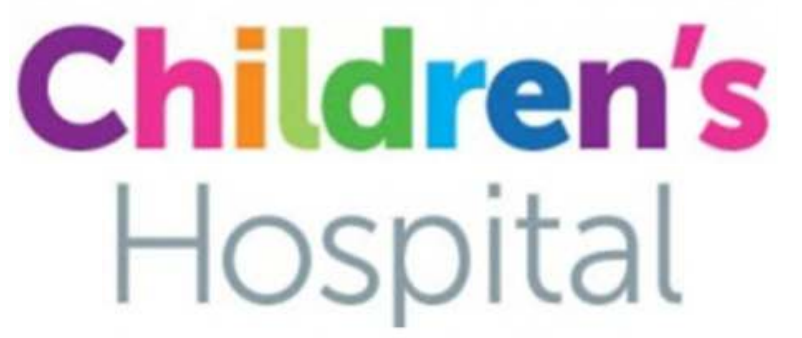

Steven Frampton ${ }^{1}$, Aria Al Ghamesi ${ }^{1}$, Robert Isaac ${ }^{1}$, Toby Pitts-Tucker ${ }^{1}$, Carolyn Way ${ }^{1}$, David Allen ${ }^{1}$, Kate Heathcote $^{2}$, Jonathan Buckland ${ }^{3}$, Hasnaa Ismail-Koch ${ }^{1}$, Andrea Burgess ${ }^{1}$

\section{Introduction}

Juvenile rheumatoid arthritis (JRA) is a paediatric auto-immune condition affecting c1:1000 children per year. Unlike adult rheumatoid arthritis, where cricoarytenoid joint fixation is common (up to $30 \%$ of patients), involvement of the cricoarytenoid joints in JRA is extremely rare. Vocal cord fixation can present in children with dysphonia, stridor, or swallowing difficulties but may initially be misdiagnosed as common pathologies such as croup.
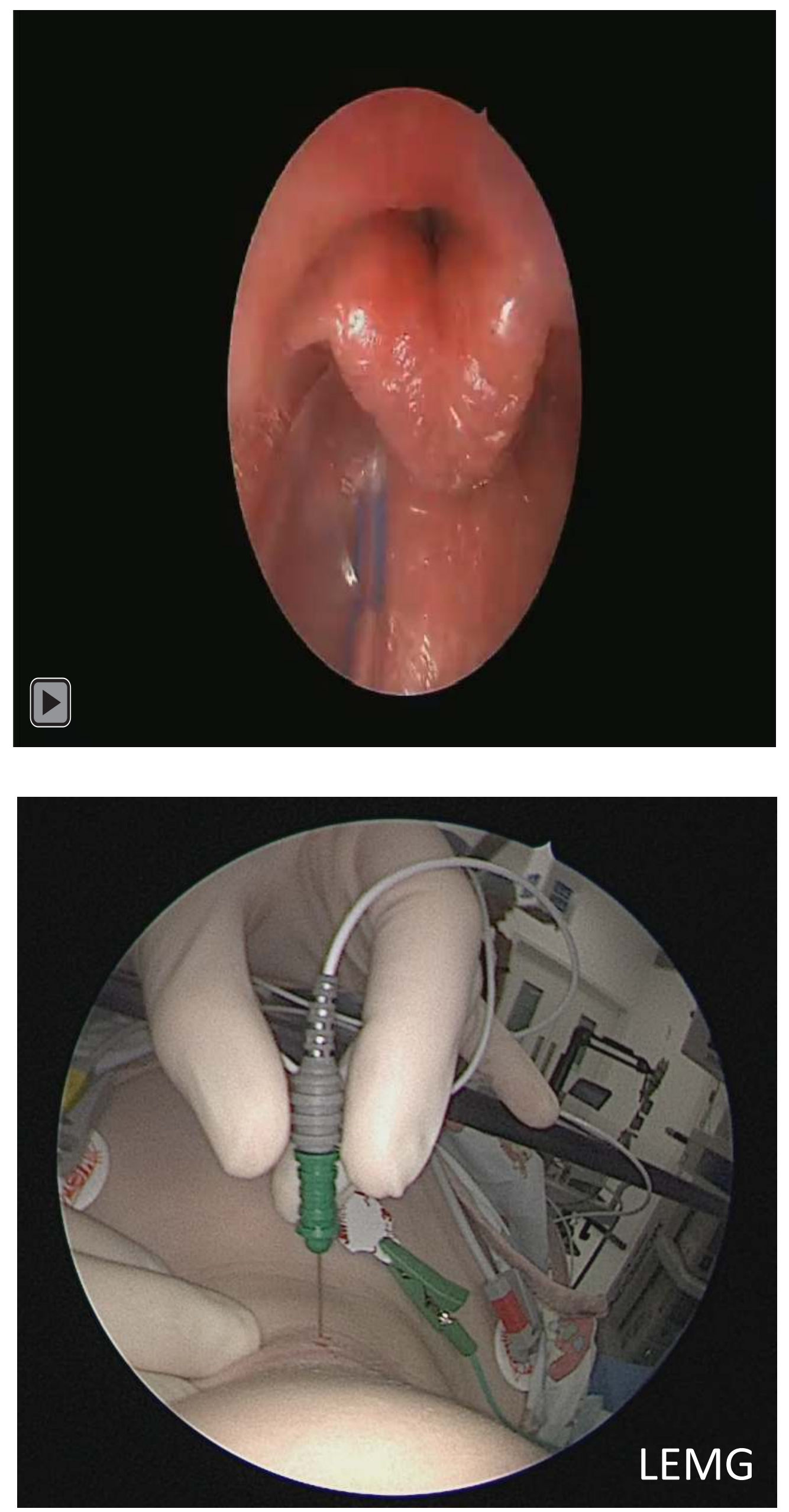

\section{Case}

A previously well $2 \frac{1}{2}$ year old girl developed progressive stridor over 5-6/52. Following unsuccessful medical management she was intubated. Following interval extubation she developed increased nocturnal work of breathing. Rigid laryngotracheobronchoscopy (LTB) revealed a soft grade I subglottic stenosis splinting the cords, with reduced cord movement and an oedematous supraglottis.

Systemic steroids produced a complete recovery but she experienced multiple minor relapses requiring steroid treatment and in-hospital stays. Outpatient flexible laryngoscopy revealed intermittent reduced vocal cord mobility but MRI of the brain and laryngeal nerves and neurology assessment were unremarkable.

Repeat LTB with laryngeal electromyography (LEMG) revealed reduced mobility of the cricoarytenoid joints but visible movement within, and action potentials from, the posterior cricoarytenoid (PCA) muscles. Preoperatively she also developed a painful knee joint. Both knee and cricoarytenoid joints were injected intraoperatively with steroid and systemic steroids were converted over time to etanercept and methotrexate. She remains relapse-free after 18 months.

\section{Conclusions}

Cricoarytenoid arthritis can be steroid-responsive, mimicking croup. Cricoarytenoid joint palpation and LEMG can be diagnostically helpful while treatment with steroids / immune modulators can avoid tracheostomy.

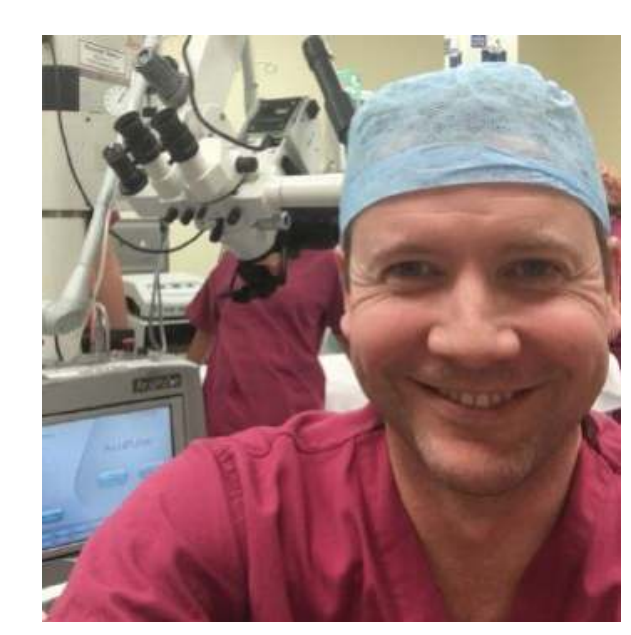

$\underset{\text { NHS Trust }}{\text { Poole Hospital WHS }}$

Portsmouth Hospitals NHS Trust

\section{University Hospital Southampton

